\title{
Special Issue on Articulated Mobile Robots (AMR)
}

\author{
Maciej Marcin Michałek • Grzegorz Granosik
}

Received: 16 November 2013 / Accepted: 20 November 2013 / Published online: 15 December 2013

(C) The Author(s) 2013. This article is published with open access at Springerlink.com

With great pleasure we would like to present the special issue devoted to Articulated Mobile Robots (AMR). The area of AMR, characterized by the combination of mobility with articulation property, still remains an attractive field of research and engineering. This substantial interest is motivated on one hand by fascination with a complicated nature of multi-body mechanisms, on the other hand by their considerable application potential, especially for the tasks where the key role play such features like agile maneuverability, dexterous manipulability, redundancy, adaptation to changing motion conditions, or flexibility and efficiency of transportation. Therefore AMR persistently inspire investigators with new challenges arising in the designing, modeling, motion planning, and control areas, as well as in diverse practical applications. Although the topic of AMR is wide and encompasses different kinds of systems ranging from hyper-

M. M. Michałek ( $\square)$

Chair of Control and Systems Engineering,

Poznań University of Technology (PUT),

Piotrowo 3A, 60-965 Poznań, Poland

e-mail: maciej.michalek@put.poznan.pl

G. Granosik

Institute of Automatic Control, Łódź University

of Technology, B. Stefanowskiego 18/22,

90-924 Łódź, Poland

e-mail: granosik@p.lodz.pl mobile robots, through self-reconfigurable structures, to underactuated robotic vehicles, there are interesting common theoretical and technical problems characteristic to the field. Despite substantial efforts paid by researchers in the area of AMR within the last three decades, some problems still remain unsolved, while other involve continued and more detailed investigation forced by growing practical needs. The purpose of this special issue is to address selected theoretical and application-oriented problems of AMR by publishing new scientific engineering results related to emerging as well as established directions of development in this fascinating area.

As an outcome of a peer review process a set of nine papers has been selected for this special issue. Among them, four papers are devoted to hypermobile and snake-like robots, two other ones address specific measurement problems emerging in the articulated mobile robotics, while remaining three articles are related to the topics of motion planning and control for N-trailer kinematic systems.

In the first group of papers Granosik provides an up-to-date survey on mechanical constructions and control approaches of the hypermobile robots. Tanaka and Matsuno consider the modeling and control issues for the case of head rising snake robots. A novel approach to locomotion control for a snake-like robot using the simplified central pattern generators network is proposed by Nor and Ma, while Pietrowska and 
Tchon study the dynamics and motion planning problem for the constrained non-holonomic trident snake robot.

The second group of papers includes description and experimental validation of the flexed postures measurement system for flexible mono-tread robot presented by Kinugasa and Akagi et al., whereas Almqvist and Magnusson et al. address in details the new perception system dedicated to the task of automated loading of LHD (load-haul-dump) vehicles.

In the third group, the multi-agent control system is proposed by Aranda-Bricaire and González-Sierra et al., which makes a formation of unicycle robots emulate the $\mathrm{N}$-trailer kinematics. Modeling and motion planning problems for a 3D generalization of the $\mathrm{N}$ trailer vehicle, in the form of the so-called multi-bar system, are considered by Jakubiak and Ratajczak. Finally, Michałek presents the formal analysis, comparison, and experimental verification of active and passive lining-up control strategies for the $\mathrm{N}$-trailer vehicles.

We would like to cordially thank Prof. Kimon Valavanis, Editor-In-Chief of JINT, for the opportunity to organize this special issue, for the confidence entrusted to us, and for his favor and support. We also kindly thank Marie Veth Chua from the Springer team for our fruitful cooperation. Finally, we would like to express our gratitude to all the Reviewers engaged in the peer review process who helped us keep high quality standards imposed by JINT. Let us mention all of them appreciating their efforts (the list in the alphabetical order):

Begoña C. Arrue, Moteaal Asadi, Paolo Bolzern, Jordan H. Boyle, Fernando Gómez Bravo, YangQuan Chen, David J. Christensen, Thomas Collins, Rongxin Cui, Brian J. Driessen, R. S. Ferguson, Edwardo F. Fukushima, Octavio Garcia, Alfonso J. GarcíaCerezo, Kyle Gilpin, Yasunobu Hitaka, Christian Holden, Gianluca Ippoliti, Masato Ishikawa, Janusz Jakubiak, Haruhisa Kurokawa, Patrick Labenda, Guoyuan Li, Shunjie Li, Anthony Mandow, Jorge L. Martínez, Takayuki Matsuo, Garvin Miller, Santhakumar Mohan, Theresa Rienmüller, TaeWon Seo, Piotr Skrzypczyński, Motoyasu Tanaka, Hiroaki Yamaguchi, Shumei Yu, Josue E. Zarate, Kai Zhang, Luis A. Zuniga Aviles.

We hope this special issue will be interesting and inspiring for the readers.

$$
\begin{aligned}
& \text { With kind regards - Guest Editors, } \\
& \text { Maciej M. Michałek, Ph.D. Eng. } \\
& \text { Grzegorz Granosik, Dc.S. Eng. }
\end{aligned}
$$

Open Access This article is distributed under the terms of the Creative Commons Attribution License which permits any use, distribution, and reproduction in any medium, provided the original author(s) and the source are credited. 